Bull. Austral. Math. Soc.

$54 \mathrm{H} 20,58 \mathrm{~F} 03$

VOL. 59 (1999) [181-186]

\title{
CHAIN RECURRENT POINTS OF A TREE MAP
}

\author{
Tao Li and Xiangdong Ye
}

\begin{abstract}
We generalise a result of Hosaka and Kato by proving that if the set of periodic points of a continuous map of a tree is closed then each chain recurrent point is a periodic one. We also show that the topological entropy of a tree map is zero if and only if the $\omega$-limit set of each chain recurrent point (which is not periodic) contains no periodic points.
\end{abstract}

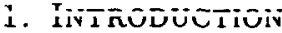

By a tree we mean a connected compact one-dimensional branched manifold containing no circle. The dynamics of a tree map, that is, a continuous map from a tree into itself, have been studied intensively in the recent years (see the references). In this paper we shall study the set of chain recurrent points of a tree map. It is known that if $f$ is an interval map and the set of periodic points of $f$ is closed, then each chain recurrent point is a periodic one [6, 13]. Recently, Hosako and Kato [5] showed that if the set of non-wandering points of a continuous map of a tree is finite, then each non-wandering point is a periodic point. We shall generalise the result of [5] and prove some other results. To be more precise, we need some notation.

A subtree of $T$ is a subset of $T$, which is itself a tree. For $x \in T$ the number of connected components of $T \backslash\{x\}$ is called the valence of $x$ in $T$. A point of $T$ of valence 1 is called an end of $T$, and a point of valence different from 2 is called a vertex of $T$. Let $V(T)$ be the set of vertices of $T$. The closure of each connected component of $T \backslash V(T)$ is called an edge of $T$. The set of ends of $T$ and the number of ends of $T$ will be denoted by $E(T)$ and End $(T)$ respectively. Let $n \geqslant 2$. A tree is said to be an $n$-star if $T$ has a point $b$ of valence $n$ and the closure of each connected component of $T \backslash b$ is an interval. Let $A \subset T$. We shall use $[A]$ to denote the smallest closed connected subset containing $A$. If $A=\{a, b\}$ then we use $[a, b]$ to denote $[A]$. We define $(a, b)=[a, b] \backslash\{a, b\}$ and we similarly define $(a, b]$ and $[a, b)$. For a subset $A$ of $T$, we use $\operatorname{int}(A), \bar{A}$ and $b(A)$ to denote the interior, the closure and the boundary of $A$ respectively.

Let $f$ be a tree map. The set of periodic points of $f$, the set of almost periodic points of $f$, the set of recurrent points of $f$, the $\omega$-limit set of $x$, the set of

Received 27th May, 1998

Project supported by NSFC 19625103.

Copyright Clearance Centre, Inc. Serial-fee code: 0004-9729/99 \$A2.00+0.00. 
non-wandering points of $f$ and the set of chain recurrent points of $f$ will be denoted by $P(f), A P(f), R(f), \omega(x, f), \Omega(f)$ and $C R(f)$ respectively (see [3] for the definitions). It is known that $P(f) \subset A P(f) \subset R(f) \subset \bigcup_{x \in T} \omega(x, f) \subset \Omega(f) \subset C R(f)$. For the notion of no division for a periodic orbit of a tree map, the notion of topological entropy of $f$ (denoted by $h(f)$ ) and the notion of minimal set see $[1,11,9,12]$. It is known that if $A$ is a minimal set of $f$, then $A \subset A P(f)$. For $x \in T$, let $\alpha(x, f)=\left\{y \in T:\right.$ there are $n_{i} \longrightarrow \infty, y_{i} \in f^{-n_{i}}(x)$, with $\left.y_{i} \longrightarrow y\right\}$.

Now we are in the position to state the main results of the paper.

THEOREM A. Let $T$ be a tree and $f: T \longrightarrow T$ be continuous. Then $C R(f)=P(f)$ if and only if $\overline{P(f)}=P(f)$.

THEOREM B. Let $f: T \longrightarrow T$ be a continuous map from a tree $T$ into itself. Then $f$ has zero topological entropy if and only if for each $x \in C R(f) \backslash P(f), \omega(x, f) \cap P(f)=\emptyset$. REMARK. As there is a continuous map $f$ from a graph $G$ into itself such that $\Omega(f)=$ $\{e, t\}$ with $f(e)=e$ and $t \notin P(f)[2]$, the conclusions of Theorem A,B do not hold for graph maps.

\section{Proofs of the Main Results}

In this section we shall give the proofs of Theorem $A$ and $B$. To do this we need the following known results.

LEMma 2.1. $[10,11,1]$ Let $T$ be a tree and $f: T \longrightarrow T$ continuous. Then

1. $\overline{P(f)}=\overline{R(f)}$.

2. $f$ has a non-divisible periodic orbit if and only if there are some $x \in T$ and some $n \in \mathbb{N}$ with $(n, m)=1$ for each $2 \leqslant m \leqslant \operatorname{End}(T)$ such that $x \in\left(f^{n}(x), f(x)\right)$.

3. $h(f)>0$ if and only there is $n \in \mathbb{N}$ such that $f^{n}$ has a non-divisible periodic orbit.

LEMMA 2.2. [8] Let $f: T \rightarrow T$ be a continuous map of a tree $T$. Then $h(f)>0$ if and only if there are some $n \in \mathbb{N}$ and two disjoint closed intervals $J_{1}, J_{2}$ contained in some edge of $T$ such that $f^{n}\left(J_{i}\right) \supset J_{1} \cup J_{2}$ for $i=1,2$.

The following two lemmas will be used in the proof of Lemma 2.5.

LEMMA 2.3. Let $T$ be a compact metric space with metric $d$ and $f: T \longrightarrow T$ be continuous. If $A$ is an open subset of $T$ such that $f(\bar{A}) \subset A$, then for each $x \in C R(f) \backslash A$ and each $n \in \mathbb{N}$ we have $f^{n}(x) \notin A$.

Proof: As $C R\left(f^{n}\right)=C R(f)$ for each $n \in \mathbb{N}$ we only need to show that $f(x) \notin A$ for each $x \in C R(f) \backslash A$. Assume the contrary. That is, there is $x \in C R(f) \backslash A$ such that $f(x) \in A$. Let

$$
\varepsilon=\inf \{d(y, z): y \in T \backslash A, z \in f(\bar{A})\}
$$


By our assumption, $\varepsilon>0$. As $x \in C R(f)$ there are $x_{0}, x_{1}, \ldots, x_{n}$ such that $x_{0}=x_{n}=x$ and $d\left(f\left(x_{i}\right), x_{i+1}\right)<\varepsilon$ for each $i=0, \ldots, n-1$. As $f\left(x_{0}\right)=f(x) \in A$ we have $x_{1} \in A$ and inductively we have $x_{i} \in A$ for $1 \leqslant i \leqslant n$. That is, $x \in A$, a contradiction.

Lemma 2.4. Let $n \in \mathbb{N}$ and $n \geqslant 2$. Then there is $l(n) \in \mathbb{N}$ such that for every $m \in \mathbb{N}$ there is $m^{\prime} \in\{m+1, \ldots, m+l(n)\}$ such that $\left(m^{\prime}, i\right)=1$ for each $2 \leqslant i \leqslant n$.

Proof: The lemma can be checked by taking $l(n)=n$ !.

The key lemma for the proofs of the main theorems will be the following. Note that we use $F(f)$ to denote the set of fixed points of $f$.

Lemma 2.5. Let $T$ be a tree and $f: T \longrightarrow T$ be continuous. If there is an $x \in C R(f) \backslash P(f)$ such that $\omega(x, f) \cap F(f) \neq \emptyset$, then $h(f)>0$.

Proof: Let $x \in C R(f) \backslash P(f)$ and $e \in \omega(x, f) \cap F(f)$.

(A) if there are $x_{1} \in(x, e)$ and some $n \in \mathbb{N}$ such that $f^{n}\left(x_{1}\right)=x$ then $h(f)>0$.

As $f^{n}\left[x_{1}, e\right] \supset[x, e]$, there is $x_{2} \in\left(x_{1}, e\right)$ with $f^{n}\left(x_{2}\right)=x_{1}$. Inductively, for each $i \geqslant 3$ there is $x_{i} \in\left(x_{i-1}, e\right)$ with $f^{n}\left(x_{i}\right)=x_{i-1}$. Since $e \in \omega(x, f)$ we have that $e \in \omega\left(x, f^{n}\right)$. Let $S$ be the component of $T \backslash\left\{x_{l(n)}\right\}$ which contains $x$. Then there is $m \in \mathbb{N}$ such that $f^{m n}(x) \notin S$. We have:

$$
x_{i} \in\left(f^{n}\left(x_{i}\right), f^{(m+i) n}\left(x_{i}\right)\right), \quad i=1,2, \ldots, l(n) .
$$

By Lemma 2.4 we know that there is $1 \leqslant i_{0} \leqslant l(n)$ such that $\left(m+i_{0}, j\right)=1$ for each $j=2, \ldots$, End $(T)$. By Lemma 2.1, $h\left(f^{n}\right)>0$, and hence $h(f)>0$.

Let $T_{1}$ be the component of $T \backslash\{x\}$ containing $e$ and assume that for each $n \in \mathbb{N}$ there is no $y \in(x, e)$ with $f^{n}(y)=x$.

(B) There is $y \in T_{1}$ such that $f(y)=x$.

Assume the contrary. That is, there is no $y \in T_{1}$ such that $f(y)=x$. Then $f\left(\overline{T_{1}}\right) \subset T_{1}$, contradicting Lemma 2.3 as $e \in T_{1}$ and $e \in \omega(x, f)$.

Let $n \in \mathbb{N}$ and $W_{n}=T_{1} \cap\left(\bigcup_{j=1}^{n} f^{-j}(x)\right)$. Let $V_{n}$ be the component of $T_{1} \backslash W_{n}$ containing $e$. As for each $n \in \mathbb{N}$ there is no $y \in(x, e)$ with $f^{n}(y)=x$, we have that $x \in \overline{V_{n}}$. Moreover, $E\left(\overline{V_{n}}\right) \subset E\left(T_{1}\right) \cup \bigcup_{j=1}^{n} f^{-j}(x)$.

(C) For each $n \in \mathbb{N}$ there is $y_{n} \in V_{n}$ such that $y_{n} \in f^{-(n+1)}(x)$.

Note that $V_{n}$ is an open subset of $T$ as $\bigcup_{j=1}^{n} f^{-j}(x)$ is closed. If there is no $y \in V_{n}$ such that $y \in f^{-(n+1)}(x)$ then we have $f\left(\overline{V_{n}}\right) \subset V_{n}$, as $e \in V_{n}$ and $T$ is uniquely arc-wise connected. This contradicts Lemma 2.3 since $e \in \omega(x, f)$ and $e \in V_{n}$.

Let $W=T_{1} \cap\left(\bigcup_{j=1}^{\infty} f^{-j}(x)\right)$ and $V$ be the component of $T_{1} \backslash W$ containing $e$. It is easy to see that $V$ contains a degenerate interval. Let $P=\bar{V} \cap \alpha(x, f)$. Then $P \subset$ $E(\widetilde{V}) \cup V\left(T_{1}\right)$ is finite. We claim: 
(D) $P$ is not empty and $f(P) \subset P$.

If $P=\emptyset$, then $V=V_{n}$ for some $n \in \mathbb{N}$. By (C), there is $y_{n} \in V$ such that $y_{n} \in f^{-n+1}(x)$, a contradiction.

Let $y \in P$. Then $f(y) \in \alpha(x, f)$ and there are $y_{n_{i}} \in f^{-n_{i}}(x)$ such that $y_{n_{i}} \longrightarrow y$ and $y_{n_{i}} \notin V$. Assume that $f(y) \notin \bar{V}$. Then there is $p \in P$ such that $p \in(e, f(y))$. If $p \in \bigcup_{j=1}^{\infty} f^{-j}(x)$, then there is $z \in(y, e)$ such that $f(z)=p$. This implies that $z \in$ $\bigcup_{j=1}^{\infty} f^{-j}(x)$, and hence $y \notin \bar{V}$, a contradiction. Thus we have $p \notin \bigcup_{j=1}^{\infty} f^{-j}(x)$. If $(p, f(y)) \cap$ $\left(\bigcup_{j=1}^{\infty} f^{-j}(x)\right) \neq \emptyset$, then there is $z \in(y, \epsilon)$ such that $f(z)=p$. This implies that $y \notin$ $\bar{V}$, a contradiction. We must have $(p, f(y)) \cap\left(\bigcup_{j=1}^{\infty} f^{-j}(x)\right)=\emptyset$. Thus $f(y) \in \bar{V}$, a contradiction. Hence $f(y) \in \bar{V} \cap \alpha(x, f)$. That is, $P$ is invariant under $f$.

As $P$ is a finite invariant subset, there is $n$ such that $f^{n}(y)=y$ for each $y \in P$. Let $g=f^{n}$ and $y_{1} \in P$. Then there are $y_{n_{i}} \in f^{-n_{i}}(x)$ with $y_{n_{i}} \longrightarrow y_{1}$. It is easy to see that there is $1 \leqslant i_{0} \leqslant n$ such that $f^{i}\left(y_{1}\right) \in f^{-n_{i}+i_{0}}(x)$ and $-n_{i}+i_{0} \mid n$. Let $y=f^{i}\left(y_{1}\right)$. Then $y \in \alpha(x, g)$ and $g(y)=y$. We have:

(E) There are $z \in T, m \geqslant 2$ with $(m, i)=1$ for each $2 \leqslant i \leqslant \operatorname{End}(T), 1 \leqslant t \leqslant \operatorname{Val}(y)$ such that $z \in\left(g^{t}(z), g^{t m}(z)\right)$.

Let $U$ be a small connected neighbourhood of $y$ such that $\bar{U}$ is homeomorphic to some $n$-star with $n=\operatorname{Val}(y)$ and $x \notin U$. Let $b_{1}, \ldots, b_{n}$ be the connected components of $U \backslash\{y\}$. As $y$ is a fixed point of $g$, there is a small connected neighbourhood $V$ of $y$ such that $g^{i}(V) \subset U$ for $i=0,1, \ldots, n+1$. Since $y \in \alpha(x, g)$ there is $n_{i}$ such that $y_{n_{i}} \in g^{-n_{i}}(x) \cap V$. Then there is $q \in V$ such that $q, g(q), \ldots, g^{t}(q) \in U$ with $q \in\left(y, g^{t}(q)\right) \subset b_{n_{0}}$ and $g^{t}(q) \in O r b\left(y_{n_{i}}, g\right)$ for some $1 \leqslant t \leqslant n, 1 \leqslant n_{0} \leqslant n$. Then we have $q_{i+1} \in b_{n_{0}}$ such that $q_{i+1} \in\left(y, q_{i}\right)$ and $g^{t}\left(q_{i+1}\right)=q_{i}$ for each $i \in \mathbb{N}$. (Set $q=q_{1}$.) By using the same idea as in the proof of $(\mathrm{A})$ and the fact that $e \in \omega(x, g)$ we get the conclusion of $(\mathrm{E})$.

By Lemma 2.2 we have $h(g)=\left(h\left(g^{t}\right)\right) / t>0$, and a consequently $h(f)>0$.

Corollary 2.6. Let $f: T \longrightarrow T$ be a continuous map from a tree $T$ into itself. If $C R(f) \neq P(f)$, then $A P(f) \neq P(f)$ and consequently, $P(f)$ is not closed.

Proof: If $h(f)>0$, then by Lemma 2.2 there are two disjoint closed intervals $J_{1}, J_{2}$ contained in some edge $E$ of $T$ and $n \in \mathbb{N}$ such that $f^{n}\left(J_{1}\right) \cap f^{n}\left(J_{2}\right) \supset J_{1} \cup J_{2}$. Hence there are a closed invariant (under $f^{n}$ ) subset $X$ of $E$ and a continuous surjective map $\phi$ : $X \longrightarrow \Sigma_{2}$ such that $\phi \circ f^{n}=f^{n} \circ \sigma$, where $\left(\Sigma_{2}, \sigma\right)$ is the one-sided shift with two symbols. Moreover, $\phi$ is one-to-one except on a countable subset of $X$ (see [3]). Then using [12] we get a non-trivial minimal set of $f^{n}$. That is, $A P\left(f^{n}\right) \neq P\left(f^{n}\right)$ and hence $A P(f) \neq P(f)$. Now we assume that $h(f)=0$ and $x \in C R(f) \backslash P(f)$. If $\omega(x, f) \cap P(f) \neq \emptyset$, then there is $y \in \omega(x, f) \cap P(f)$ with period $n$ for some $n \in \mathbb{N}$. Thus there is $i \in \mathbb{N}$ such that 
$f^{i}(y) \in \omega\left(x, f^{n}\right)$ as $\omega(x, f)=\bigcup_{i=1}^{n} \omega\left(f^{i}(x), f^{n}\right)$ and $f\left(\omega\left(f^{j}(x), f^{n}\right)\right)=\omega\left(f^{j+1}(x), f^{n}\right)$ for each $j \in \mathbb{N}$. Note that $f^{i}(y)$ is a fixed point of $f^{n}$. By Lemma 2.5, we have $h\left(f^{n}\right)>0$, a contradiction. Hence we have $\omega(x, f) \cap P(f)=\emptyset$. Let $A$ be a minimal set contained in $\omega(x, f)$. Then $A$ is not trivial. That is, $A P(f) \neq P(f)$.

As $P(f) \subset A P(f) \subset \overline{R(f)}=\overline{P(f)}$, we have that $P(f)$ is not closed.

Proof of Theorem A: It is clear $C R(f)=P(f)$ implies $P(f)$ is closed. Now assume that $P(f)$ is closed. By Corollary 2.6, we have $C R(f)=P(f)$. This ends the proof.

To prove Theorem B we need the following lemma.

LEMma 2.7. Let $f: T \longrightarrow T$ be a continuous map from a tree $T$ into itself. If $h(f)>0$, then there is $x \in \mathcal{L} K(f) \backslash \mu(f)$ such that $f^{\prime \prime}(x) \in H(f)$ tor some $n \in \mathbb{N}$.

Proof: By Lemma 2.2 there are two disjoint closed intervals $J_{1}, J_{2}$ contained in some edge $E$ of $T$ and $n \in \mathbb{N}$ such that $f^{n}\left(J_{1}\right) \cap f^{n}\left(J_{2}\right) \supset J_{1} \cup J_{2}$. Let $g=f^{n}, J_{i}=\left[a_{i}, b_{i}\right]$ and give an orientation of $E$ such that for each $x_{i} \in J_{i}, x_{1}<x_{2}$. Then there are a fixed point $e \in J_{1}$ of $g$ and $z \in J_{1}$ such that $g(z)=b_{2}$. Without loss of generality we assume that $e<z$ and $(e, z) \cap F(g)=\emptyset$. Take a point $z<w \in E$ such that $g(w)=e$. Then $w \notin P(f)$ and $w \in \Omega(g) \subset C R(f)$. Hence $w \in C R(f) \backslash P(f)$ and $f^{n}(w) \in P(f)$.

Proof of Theorem B: Assume that $h(f)=0$ and there is $x \in C R(f) \backslash P(f)$ with $\omega(x, f) \cap P(f) \neq \emptyset$. Let $y \in \omega(x, f) \cap P(f)$ and let the period of $y$ be $n$. As $\omega(x, f)=\bigcup_{i=0}^{n-1} \omega\left(f^{i}(x), f^{n}\right)$, there is $i$ such that $y \in \omega\left(f^{i}(x), f^{n}\right)$. Hence $f^{n-i}(y) \in$ $\omega\left(x, f^{n}\right)$. Note that $f^{n-i}(y)$ is a fixed point of $f^{n}$ and $x \in C R(f) \backslash P(f)=C R\left(f^{n}\right) \backslash P\left(f^{n}\right)$, a contradiction.

The sufficiency of the theorem follows from Lemma 2.7.

\section{REFERENCES}

[1] L. Alseda and X. Ye, 'No-division and the set of periods for tree maps', Ergodic Theory Dynamical Systems 15 (1995), 221-237.

[2] L. Block, 'Continuous maps of the interval with finite nonwandering set', Trans. Amer. Math. Soc. 240 (1978), 221-230.

[3] L. Block and W.A. Coppel, Dynamics in one dimension, Lect. Notes in Math. 1513 (Springer-Verlag, Berlin, Heidelberg, New York, 1992).

[4] J. Franks and M. Misiurewicz, 'Cycles for disk homeomorphisms and thick trees', Contemp. Math. 152 (1993), 69-139.

[5] H. Hosaka and H. Kato, 'Continuous maps of trees and non-wandering sets', Topology Appl. 81 (1997), 35-46.

[6] Z. Nitecki, 'Maps of the interval with closed periodic set', Proc. Amer. Math. Soc. 85 (1982), 451-456.

[7] T. Li, M. Misiurewicz, G. Pianigian and J. Yorke, 'Odd chaos', Physics Lett. A 87 (1981), 271-273. 
[8] J. Llibre and M. Misiurewicz, 'Horseshoe, entropy and periods for graph maps', Topology 32 (1993), 649-664.

[9] P. Walters, An introduction to ergodic theory (Springer-Verlag, Berlin, Heidelberg, New York, 1982).

[10] X. Ye, 'The center and the depth of the center of a tree map', Bull. Austral. Math. Soc. 48 (1993), 347-350.

[11] X. Ye, 'Tree maps with non divisible periodic orbits', Bull. Austral. Math. Soc. 56 (1997), 467-471.

[12] X. Ye, 'D-function of a minimal set and an extension of Sharkovskii' theorem', Ergod. Theory Dynamical Systems 12 (1992), 365-376.

[13] Z. Zhou, 'Self-mappings of the interval without homoclinic points', (in Chinese), Acta. Math. Sinica 25 (1982), 633-640.

[14] J. Zhang and J. Xiong, Iterations of functions and one-dimensional dynamics, (in Chinese) (Sichuan Education Press, 1992).

Department of Mathematics

University of Science and Technology of China

Hefei, Anhui 230026

Peoples Republic of China

e-mail: yexd@ustc.edu.cn 